\title{
Matrine in association with FD-2 stimulates F508del-cystic fibrosis transmembrane conductance regulator activity in the presence of corrector VX809
}

\author{
BARBARA MARENGO ${ }^{1}$, ANDREA SPECIALE ${ }^{1}$, LISA SENATORE ${ }^{1}$, SILVANO GARIBALDI $^{2}$, \\ FRANCESCA MUSUMECI $^{3}$, ERIKA NIEDDU ${ }^{3}$, BENEDETTA POLLAROLO $^{3}$, MARIA ADELAIDE PRONZATO $^{1}$, \\ SILVIA SCHENONE ${ }^{3}$, MAURO MAZZEI ${ }^{3}$ and CINZIA DOMENICOTTI ${ }^{1}$ \\ ${ }^{1}$ Department of Experimental Medicine; ${ }^{2}$ Division of Cardiology, IRCCS University Hospital San Martino, \\ Research Centre of Cardiovascular Biology; ${ }^{3}$ Department of Pharmacy, University of Genoa, I-16132 Genoa, Italy
}

Received February 28, 2017; Accepted July 27, 2017

DOI: $10.3892 / \mathrm{mmr} .2017 .7736$

\begin{abstract}
Cystic fibrosis is caused by mutations of the cystic fibrosis transmembrane conductance regulator (CFTR) gene, and the predominant mutation is termed Phe508del (F508del). Therapy for F508del-CFTR patients is based on the use of Orkambi $^{\circledR}$, a combination of VX809 and VX770. However, though Orkambi leads to an improvement in the lung function of patients, a progressive reduction in its efficacy has been observed. In order to overcome this effect, the aim of the present study was to investigate the role of matrine and the in-house compound FD-2 in increasing the action of VX809 and VX770. Fischer rat thyroid cells overexpressing F508del-CFTR were treated with matrine, VX809 (corrector) and/or with a number of potentiators (VX770, FD-1 and FD-2). The results demonstrated that matrine was able to stimulate CFTR activity and, in association with FD-2, increased the functionality of the channel in the presence of VX809. Based on these results, it may be hypothesized that FD-2 may be a novel and more effective potentiator compared with VX770.
\end{abstract}

\section{Introduction}

Cystic fibrosis (CF) is an autosomal recessive genetic disorder caused by mutations of the gene that encodes the cystic fibrosis transmembrane conductance regulator (CFTR) protein (1). This protein is a chloride (or bicarbonate)-conducting anion channel expressed on the membrane of epithelial cells of the

Correspondence to: Professor Cinzia Domenicotti, Department of Experimental Medicine, University of Genoa, 2L.B. Alberti Street, I-16132 Genoa, Italy

E-mail: cinzia.domenicotti@unige.it

Abbreviations: CF, cystic fibrosis; CFTR, cystic fibrosis transmembrane conductance regulator; FRT, Fischer rat thyroid

Key words: CF, CFTR, matrine, VX809, VX770, FD-2, FRT cells lung, intestines and pancreas, which modulates electrolytic exchange. Therefore, mutations in CFTR, leading to altered ion transport, induce a progressive deterioration of the target organs and, consequently, premature mortality of patients (2). CFTR mutations may induce alterations in maturation, subcellular localization (trafficking) and activity (gating) of the CFTR protein $(3,4)$. The predominant CFTR mutation is termed Phe508del (F508del), and results in severely impaired protein maturation with a consequent alteration of CFTR membrane translocation $(5,6)$. However, in the case that the F508del-CFTR is able to reach the plasma membrane, it exhibits reduced activity and stability in the membrane, in addition to a greater tendency towards degradation by the ubiquitin/proteasome system $(7,8)$.

Research has primarily focused on the identification and development of modulators that are able to resolve gating and trafficking problems (9). The 'corrector' acts on trafficking and promotes CFTR membrane localization $(10,11)$ while the 'potentiator' acts on gating and leads to an increase in CFTR activity (12). Therefore, any therapy should be designed by choosing the most appropriate modulator which is able to target the specific mutation. However, in patients with F508del-CFTR, it has been demonstrated that treatment with a potentiator is ineffective, since it is unable to induce CFTR maturation and membrane translocation. Therefore, in these patients, the co-administration of a corrector and a potentiator is necessary to allow for the almost complete repair of CFTR trafficking and gating (13).

At present, there are two molecules which are frequently used: The potentiator VX770 (ivacaftor) and the corrector VX809 (lumacaftor). Recently, the use of Orkambi ${ }^{\circledR}$, which is a combination of VX770 and VX809, was approved (3). However, though an improvement in the quality of life of patients treated with the Orkambi was observed, the drug induced a modest improvement in lung function (14). This limited effect may be due to an inhibitory action exerted by VX770 on VX809 (15). In order to overcome this problem, research is focusing on the analysis of natural molecules that may resolve the problem at its origin; among them, matrine, an alkaloid extracted from roots of Sophora flavescens, appears to provide promising results. In 
particular, matrine, by interacting with the heat shock cognate (HSC)/heat shock $70 \mathrm{kDa}$ protein 1A (HSP70) chaperone system, led to an increase in F508del-CFTR membrane localization (16), and the corrector activity of matrine was observed at high doses (0.4-0.8 mM) which may be toxic for long-term treatment of patients with $\mathrm{CF}$.

The aim of the present study was to investigate whether lower concentrations $(30 \mu \mathrm{M})$ of matrine (PubChem CID: 91466) may be able to render more effective the action of the known corrector VX809 (PubChem CID: 16678941) and the potentiator VX770 (PubChem CID: 16220172), in addition to that of two dihydropyridines, FD-1 (F508 act $^{-05)}$ (17) and FD-2, the latter being a newly-synthesized compound. The use of dihydropyridines may be advantageous, since these molecules were established as potentiators with notable activity, simple synthesis and a low production cost, by Pedemonte et al (18), Cateni et al (19) and Giampieri et al (20). Due to the cited interference between VX770 and VX809, it was deemed noteworthy to investigate the behavior of this class of potentiators, coupled with the corrector VX809. Therefore, FD-1, a compound with a moderate level of activity against FD-2, was selected. A previous study on asymmetrical dihydropyridines demonstrated the ability of the benzyl group to maximize potentiator activity (20), which provided the basis for the analysis of a newly-synthesized dihydropyridine (FD-2) bearing two benzyl groups at the ester level.

\section{Materials and methods}

Cell culture and treatments. Fischer rat thyroid (FRT) cells, stably transfected with F508del-CFTR and yellow fluorescent protein (YFP), were provided by Dr L.J. Galietta (G. Gaslini Institute, Genoa, Italy). The co-expressed YFP acts as a halide sensitive dye that may be utilized to measure the anion permeability of CFTR $(18,21)$. Cells were cultured in Coon's modified F-12 medium (Sigma-Aldrich; Merck KGaA, Darmstadt, Germany) supplemented with $5 \%$ fetal bovine serum (EuroClone SpA, Pero, Italy), $2 \mathrm{mM}$ glutamine (EuroClone $\mathrm{SpA}$ ), 1\% penicillin/streptomycin (EuroClone SpA), $0.8 \mathrm{mg} / \mathrm{ml}$ zeocin (Thermo Fisher Scientific, Inc., Waltham, MA, USA) and $1.5 \mathrm{mg} / \mathrm{ml} \mathrm{G} 418$ (Sigma-Aldrich; Merck KGaA). Cells were plated ( $1 \times 10^{5}$ cells/well) into 96 -well microplates and treated with matrine (30 $\mu \mathrm{M}$; Sigma-Aldrich; Merck KGaA) for 24, 48 and $72 \mathrm{~h}$. In a series of experiments, cells were co-treated for 24,48 or $72 \mathrm{~h}$ with $2 \mu \mathrm{M}$ VX809 (Selleck Chemicals, Houston, TX, USA) and/or with one of three potentiators: $10 \mu \mathrm{M}$ VX770 (Selleck Chemicals), $10 \mu \mathrm{M}$ FD-1 and $10 \mu \mathrm{M}$ FD-2 (both synthesized in the Department of Pharmacy, University of Genoa, Genoa, Italy).

The stock solutions of all tested compounds were prepared in dimethyl sulfoxide (DMSO) and pilot studies demonstrated that the final DMSO concentration did not alter any of the cellular responses analyzed. In addition, under all conditions, the data obtained in treated cells was compared to DMSO-treated cells (Ctr). The pilot study was performed by treating cells with the highest dose of DMSO used to dissolve all tested compounds for 24,48 and $72 \mathrm{~h}$. Then, cell viability was evaluated by MTT assay.

FD-2 was synthesized following the procedure described in Cateni et al (19). Benzyl acetoacetate, 4-isopropylbenzaldehyde

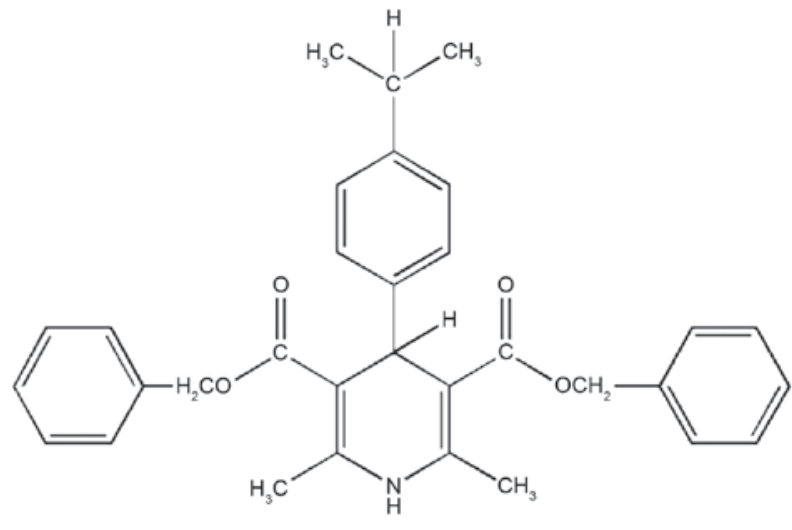

Figure 1. Structure of the FD-2 compound.

and ammonia were dissolved in isopropanol and refluxed for $4 \mathrm{~h}$. Subsequently, the crude product was subjected to chromatography on a silica gel (n-hexane/diethylether) and the residue was crystallized from cyclohexane. Yield, 25\%; melting point $107-109^{\circ} \mathrm{C}$. ${ }^{1} \mathrm{H}$-nuclear magnetic resonance $\left(\mathrm{CDCl}_{3}\right)$ : 81.22-1.27 [m, 6H, CH $\left.\left(\mathrm{CH}_{3}\right)_{2}\right] ; 2.34\left(\mathrm{~s}, 6 \mathrm{H}, \mathrm{CH}_{3}\right) ; 2.80[\mathrm{~m}$, $\left.1 \mathrm{H}, \mathrm{CH}\left(\mathrm{CH}_{3}\right)_{2}\right] ; 5.09-5.13\left(\mathrm{~m}, 5 \mathrm{H}, 2 \mathrm{CH}_{2}+\mathrm{H}-4\right) ; 5.78$ (br s, $1 \mathrm{H}$, $\mathrm{NH}$ ); and 7.04-7.32 (m, 14H Ar). Infrared (KBr): $3439(\mathrm{NH})$; and $1691(\mathrm{CO}) \mathrm{cm}^{-1}$. Combustion elemental analysis calculated for $\mathrm{C}_{32} \mathrm{H}_{33} \mathrm{NO}_{4}$ : C 77.55, H 6.71, N 2.83; observed: C 77.57, H 6.40, N 2.97 (Fig. 1).

MTT assay. Cell viability was determined using MTT (Sigma-Aldrich; Merck KGaA) staining $(22,23)$. Cells were seeded into 96-well microplates (Corning Incorporated, Corning, NY, USA) at a density of $1 \times 10^{5}$ cells/well and treated as described above. Subsequently, the cells were incubated with $0.5 \mathrm{mg} / \mathrm{ml} \mathrm{MTT}$ for $3 \mathrm{~h}$ at $37^{\circ} \mathrm{C}$. Following incubation, the supernatant was discarded, the insoluble formazan precipitates were dissolved in $\mathrm{HCl}$ (0.1 $\mathrm{M}$ in isopropanol) and the absorbance at $570 / 630 \mathrm{~nm}$ was recorded using a microplate reader (EL-808; BioTek Instruments Inc., Winooski, VT, USA).

Fluorescence assay. CFTR activity was determined using a fluorescence assay $(18,21,24)$. Cells were plated (100,000 cells/well) into black 96-well microplates with clear plastic bottoms (Corning Incorporated). Following treatment as described above, cells were washed with PBS (137 mM $\mathrm{NaCl}, 2.7 \mathrm{mM} \mathrm{KCl}, 8.1 \mathrm{mM} \mathrm{Na} \mathrm{HPO}_{4}, 1.5 \mathrm{mM} \mathrm{KH}_{2} \mathrm{PO}_{4}$, $1 \mathrm{mM} \mathrm{CaCl}_{2}$ and $0.5 \mathrm{mM} \mathrm{MgCl}$; $\mathrm{pH} 7.4$ ) and stimulated for $20 \mathrm{~min}$ with $20 \mu \mathrm{M}$ forskolin. Microplates were subsequently transferred to a microplate reader (TECAN Infinite ${ }^{\circledR}$ F200 PRO; Tecan Group, Ltd., Männedorf, Switzerland) equipped with excitation $(485 \pm 20 \mathrm{~nm})$ and emission $(535 \pm 25 \mathrm{~nm})$ filters. Each assay consisted of a continuous $14 \mathrm{sec}$ fluorescence reading with $2 \mathrm{sec}$ prior to and $12 \mathrm{sec}$ following the injection of $165 \mu \mathrm{l}$ iodide-containing PBS (PBS with $\mathrm{Cl}^{-}$replaced with $\mathrm{I}^{-}$). The $\mathrm{I}^{-}$influx rate was evaluated by calculating the variation of fluorescence intensity prior to and following the $\mathrm{I}^{-}$injection.

Data analysis. Results are expressed as the mean \pm standard error of the mean from at least three independent experiments. The statistical significance of any parametric differences 
A

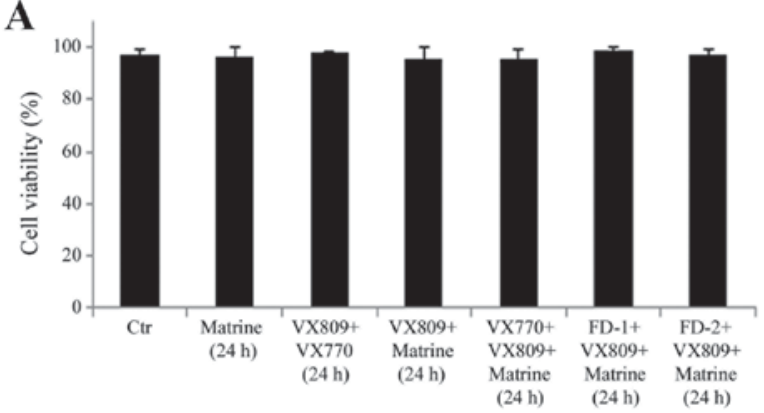

B
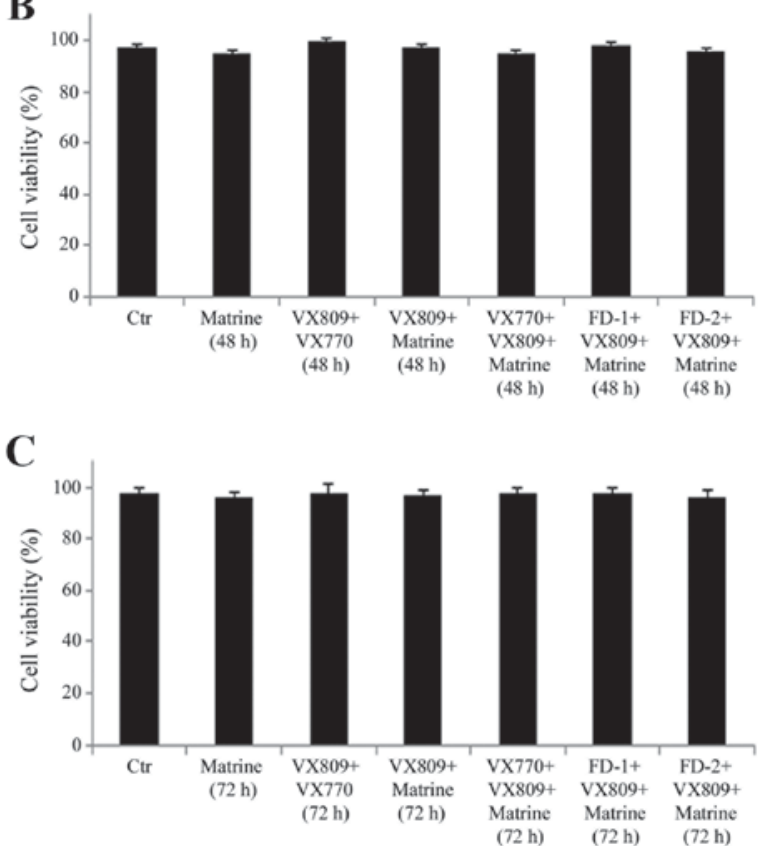

Figure 2. Matrine, VX809 or potentiators (VX770, FD-1 and FD-2), alone or in combination, do not affect the viability of F508del-CFTR FRT cells. Cell viability was evaluated in F508del-CFTR FRT cells treated with matrine $(30 \mu \mathrm{M})$ and VX809 $(2 \mu \mathrm{M})$ and/or potentiators (VX770, FD-1 and FD-2; $10 \mu \mathrm{M}$ ) for (A) $24 \mathrm{~h}$, (B) $48 \mathrm{~h}$ or (C) $72 \mathrm{~h}$. The histogram summarizes quantitative data of the mean \pm standard error of the mean of three independent experiments. FRT, Fischer rat thyroid; CFTR, cystic fibrosis transmembrane conductance regulator.

among the sets of experimental data was evaluated using one-way analysis of variance and Dunnett's test for multiple comparisons. GraphPad Prism software (version 4; GraphPad Software, Inc., La Jolla, CA, USA) was used for analysis. $\mathrm{P}<0.05$ was considered to indicate a statistically significant difference.

\section{Results}

Treatment with matrine, VX809 or potentiators (VX770, FD-1 and FD-2), alone or in combination, does not affect the viability of F508del-CFTR FRT cells. F508del-CFTR FRT cells were exposed for 24, 48 and $72 \mathrm{~h}$ to matrine, VX809, VX770, FD-1 and FD-2, alone or in combination, and MTT analysis revealed that none of the treatments was cytotoxic (Fig. 2).

Treatment with matrine alone, and in combination with VX809 plus VX770, is able to stimulate CFTR activity. As exhibited in Fig. 3A, matrine alone stimulated the activity of
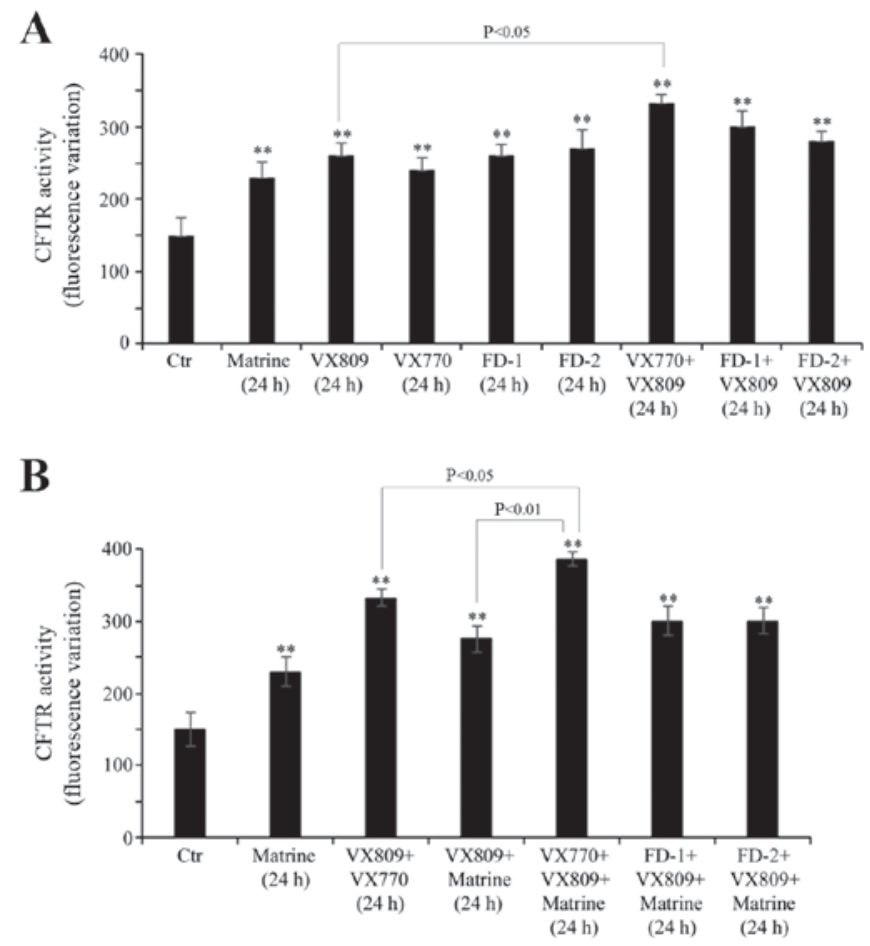

Figure 3. Matrine is able to stimulate CFTR activity, and treatment with VX809 and/or potentiators (VX770, FD-1 and FD-2) maintains its effect on CFTR activity. The activity of CFTR was analyzed in F508del-CFTR FRT cells treated for $24 \mathrm{~h}$ with matrine $(30 \mu \mathrm{M})$, VX809 $(2 \mu \mathrm{M}), \mathrm{VX} 770(10 \mu \mathrm{M})$, FD-2 $(10 \mu \mathrm{M})$ and FD-1 (10 $\mu \mathrm{M}),(\mathrm{A})$ alone or (B) in combination. Results are reported as the variation of fluorescence (fluorescence intensity prior to and following $\mathrm{I}^{-}$injection). The histogram summarizes quantitative data of the mean \pm standard error of the mean of three independent experiments. ${ }^{* *} \mathrm{P}<0.01$ vs. Ctr cells. FRT, Fischer rat thyroid; CFTR, cystic fibrosis transmembrane conductance regulator; Ctr, control.

mutant CFTR by 53\% compared with untreated cells, which was a similar result to that observed when the cells were treated with the other compounds individually. In addition, a 24-h VX809/VX770 co-treatment stimulated the effect of VX809 on CFTR activity by $30 \%$ (Fig. 3A).

In order to evaluate whether matrine was able to increase the VX809-induced CFTR activity, other experiments were performed by treating the cells for $24 \mathrm{~h}$ with a combination of matrine, VX809 and/or potentiators. As illustrated in Fig. 3B, all the combinations tested were able to stimulate CFTR activity by $80-100 \%$ compared with control cells. In addition, the presence of matrine further stimulated the effect of the VX809/VX770 combination on CFTR activity by $16 \%$.

Matrine co-treatment increases the effect of VX809 in combination with VX770. Following $48 \mathrm{~h}$ of treatment, the VX809/VX770 combination stimulated the activity of mutant CFTR by $120 \%$ compared with control cells, and the co-administration of matrine further increased CFTR activity by $25 \%$ (Fig. 4). Notably, the co-treatment of FD-1 with matrine/VX809 had a similar effect to that observed in VX809/VX770-treated cells (Fig. 4). FD-2, in combination with matrine/VX809, stimulated the activity of mutant CFTR with a similar efficiency to matrine/VX770/VX809 (Fig. 4). 


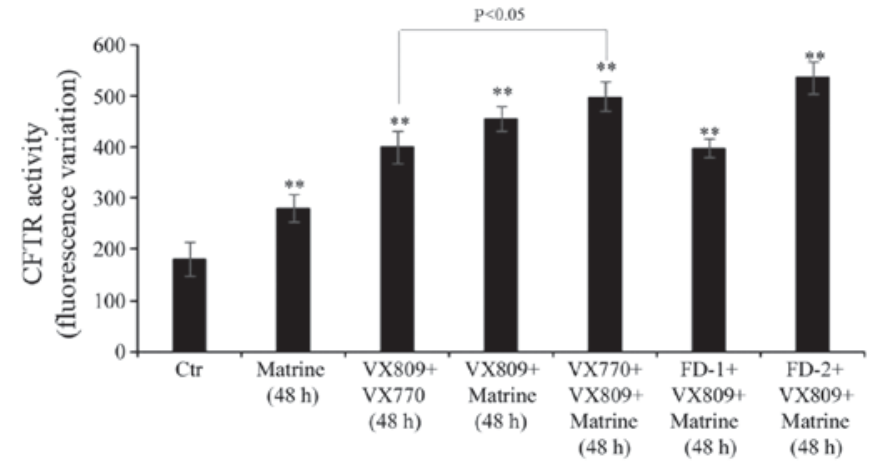

Figure 4. Matrine co-treatment for $48 \mathrm{~h}$ increases the stimulating effect of VX809 on CFTR activity. The activity of CFTR was analyzed in F508del-CFTR FRT cells treated with matrine $(30 \mu \mathrm{M})$ and VX809 $(2 \mu \mathrm{M})$ and/or potentiators (VX770, FD-1 and FD-2; $10 \mu \mathrm{M}$ ) for $48 \mathrm{~h}$. Results are reported as the variation of fluorescence (fluorescence intensity prior to and following $\mathrm{I}^{-}$injection). The histogram summarizes quantitative data of the mean \pm standard error of the mean of three independent experiments. ${ }^{* *} \mathrm{P}<0.01$ vs. Ctr cells. FRT, Fischer rat thyroid; CFTR, cystic fibrosis transmembrane conductance regulator; Ctr, control.

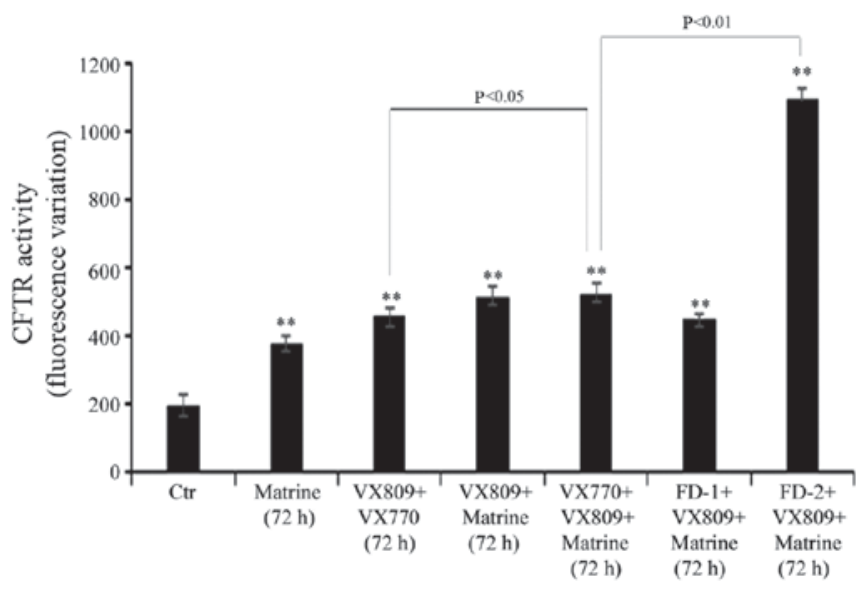

Figure 5. Combined treatment with matrine and FD-2 for $72 \mathrm{~h}$ increases the action of VX809 on CFTR activity. The activity of CFTR was analyzed in F508del-CFTR FRT cells treated with matrine $(30 \mu \mathrm{M})$ and VX809 $(2 \mu \mathrm{M})$ and/or potentiators (VX770, FD-1 and FD-2; $10 \mu \mathrm{M}$ ) for $72 \mathrm{~h}$. Results are reported as the variation of fluorescence (fluorescence intensity prior to and following $\mathrm{I}^{-}$injection). The histogram summarizes quantitative data of the mean \pm standard error of the mean of three independent experiments. ${ }^{* *} \mathrm{P}<0.01$ vs. Ctr cells. FRT, Fischer rat thyroid; CFTR, cystic fibrosis transmembrane conductance regulator; $\mathrm{Ctr}$, control.

Matrine-FD-2 co-treatment markedly increases the effect of $V X 809$. Following $72 \mathrm{~h}$ of treatment, the combination of matrine with FD-2 and VX809 stimulated mutant CFTR activity by $450 \%$ compared with control cells (Fig. 5). Notably, when VX770 was administered in place of FD-2, the rate of mutant CFTR activity was increased only by $165 \%$ compared with that observed in untreated cells (Fig. 5). However, it is necessary to note that, as already observed at $48 \mathrm{~h}$, the VX809/VX770 combination stimulated the activity of mutant CFTR by $130 \%$ (Fig. 5) and the co-administration of matrine further increased CFTR activity by $15 \%$ (Fig. 5).

In addition, the effect of FD-1 co-treatment with matrine and VX-809 produced similar effects to those observed in cells co-treated with VX770 instead of FD-1 (Fig. 5).

\section{Discussion}

$\mathrm{CF}$ is the most common autosomal recessive disease with a fatal outcome (8). The disease is characterized by alterations in the maturation and function of CFTR, a channel protein responsible for anion transport, particularly chloride ions. To date, $>2,000$ CFTR mutations have been identified, and it has been observed that consequent electrolyte imbalances lead to a progressive loss of function of a number of organs, resulting in the mortality (25). Considering the high incidence of this disease, it has become necessary to identify novel therapies and to improve the effectiveness of existing treatments. The current therapeutic approaches are primarily based on the use of antibiotics, pancreatic enzymes, anti-inflammatory drugs and mucolytics which, while improving the symptoms, do not alter the outcome of the pathology.

However, novel pharmacotherapeutic approaches are based on the use of correctors and/or potentiators. Among the known correctors, VX809 (lumacaftor) is presently used to treat patients with the F508del-CFTR mutation (a class II mutation) in which there is an alteration in CFTR trafficking (10), and acts by inducing CFTR protein maturation in the endoplasmic reticulum/Golgi apparatus and by increasing CFTR translocation to the cell membrane. Potentiators are able to counteract gating defects due to mutations, including G551D (a class III mutation). The F508del-CFTR protein, though able to translocate to the plasma membrane, is not functionally active (12), while VX770 (ivacaftor), acting as a potentiator, is able to stimulate the activity of the mutant CFTR when the mutation is class III.

In order to improve the efficacy of the therapy, a novel approach is to combine a corrector with a potentiator, and, among the new drugs, Orkambi (which consists of the combination of VX809 that facilitates F508del-CFTR maturation, and VX770 which improves its function) was the first approved therapy to treat homozygous patients for the F508del-CFTR mutation (26). In these patients, the use of a potentiator alone is ineffective as it does not stimulate CFTR trafficking, only stimulating the activity of the CFTR present in the cell membrane. However, a previous study has demonstrated that this combined approach reduces the efficacy of VX809 (15). These previous results are in line with a recent study reporting that, although Orkambi induces an improvement in symptomatology, long-term treatment leads to a progressive reduction of its pharmacological efficacy, attributed to the VX770-mediated destabilization of VX809-rescued F508del-CFTR (15). In order to reduce this inhibitory effect, other molecules with a different mechanism of action compared with that of currently-used drugs are being evaluated.

Matrine, a natural molecule used in traditional Chinese medicine, may be effective in reducing the aforementioned inhibitory effect. Matrine, interacting with the HSC/HSP70 chaperone system, downregulates the expression of HSC70 and increases the protein levels of F508del-CFTR (16). Therefore, the results of the present study are promising. Matrine was able to stimulate CFTR activity, further increase the functionality of the channel in the presence of VX809 and moderately affect the action of VX77/VX809. In particular, this action of matrine was observed at $72 \mathrm{~h}$ and may be due to the fact that this compound requires time to stimulate CFTR activity. The 
inhibitory effect of VX770 on VX809, not detectable at $24 \mathrm{~h}$, was evident at $48 \mathrm{~h}$ and at $72 \mathrm{~h}$. In addition, the results of the present study suggested that matrine may partially counteract the inhibitory effect of VX770 on VX809 under all the time conditions assayed.

Previous research has led to the development and study of novel potentiators and, among those tested, the results of the present study demonstrated that FD-2, in the presence of matrine, was able to markedly increase the CFTR activity induced by VX809. This effect was time-dependent and was particularly evident at $72 \mathrm{~h}$. The different degrees of action of matrine on VX770/VX809 and FD2/VX809 is likely to be due to the different mechanisms of action of the two potentiators. This hypothesis will be matter of investigation in our future studies.

In conclusion, the results of the present study proposed FD-2 to be a novel and possibly more efficacious potentiator, compared with VX770. Although it is necessary to perform these treatments on other cellular models and to also validate these data in in vivo systems, the present results may be useful in proposing novel and more effective therapeutic approaches in $\mathrm{CF}$.

\section{Acknowledgements}

The authors of the present study would like to acknowledge Mr. Giuseppe Catalano (DIMES, University of Genoa) for technical assistance and Ms. Suzanne Patten for language editing. The present study was supported by grants from Genoa University (B. Marengo) and funds from the Italian Cystic Fibrosis Research Foundation, Verona, Italy (M. Mazzei).

\section{References}

1. Rommens JM, Iannuzzi MC, Kerem B, Drumm ML, Melmer G, Dean M, Rozmahel R, Cole JL, Kennedy D, Hidaka N, et al: Identification of the cystic fibrosis gene: Chromosome walking and jumping. Science 245: 1059-1065, 1989.

2. Corradi V, Vergani P and Tieleman DP: Cystic fibrosis transmembrane conductance regulator (CFTR): Closed and open state channel models. J Biol Chem 290: 22891-22906, 2015.

3. Elborn JS: Cystic fibrosis. Lancet 388: 2519-2531, 2016.

4. Caputo A, Hinzpeter A, Caci E, Pedemonte N, Arous N, Di Duca M, Zegarra-Moran O, Fanen P and Galietta LJ: Mutation-specific potency and efficacy of cystic fibrosis transmembrane conductance regulator chloride channel potentiators. J Pharmacol Exp Ther 330: 783-791, 2009.

5. Cheng SH, Gregory RJ, Marshall J, Paul S, Souza DW, White GA, O'Riordan CR and Smith AE: Defective intracellular transport and processing of CFTR is the molecular basis of most cystic fibrosis. Cell 63: 827-834, 1990.

6. Dalemans W, Barbry P, Champigny G, Jallat S, Dott K, Dreyer D, Crystal RG, Pavirani A, Lecocq JP and Lazdunski M: Altered chloride ion channel kinetics associated with the delta F508 cystic fibrosis mutation. Nature 354: 526-528, 1991.

7. Riordan JR: CFTR function and prospects for therapy. Annu Rev Biochem 77: 701-726, 2008.

8. Pedemonte N and Galietta LJ: Pharmacological Correctors of Mutant CFTR Mistrafficking. Front Pharmacol 3: 175, 2012.

9. Nieddu E, Pollarolo B, Mazzei MT, Anzaldi M, Schenone S, Pedemonte N, Pesce E, Galietta LJ and Mazzei M: The search for a common structural moiety among selected pharmacological correctors of the mutant CFTR chloride channel. Futur Med Chem 6: 1857-1868, 2014.
10. Wang X, Venable J, LaPointe P, Hutt DM, Koulov AV, Coppinger J, Gurkan C, Kellner W, Matteson J, Plutner H, et al: Hsp90 cochaperone Ahal downregulation rescues misfolding of CFTR in cystic fibrosis. Cell 127: 803-815, 2006.

11. Mu TW, Ong DS, Wang YJ, Balch WE, Yates JR III, Segatori L and Kelly JW: Chemical and biological approaches synergize to ameliorate protein-folding diseases. Cell 134: 769-781, 2008.

12. Zegarra-Moran O, Monteverde M, Galietta LJ and Moran O: Functional analysis of mutations in the putative binding site for cystic fibrosis transmembrane conductance regulator potentiators. Interaction between activation and inhibition. J Biol Chem 282: 9098-9104, 2007.

13. Sondo E, Tomati V, Caci E, Esposito AI, Pfeffer U, Pedemonte N and Galietta LJ: Rescue of the mutant CFTR chloride channel by pharmacological correctors and low temperature analyzed by gene expression profiling. Am J Physiol Cell Physiol 301: C872-C885, 2011.

14. Langron E, Simone MI, Delalande CM, Reymond JL, Selwood DL and Vergani P: Improved fluorescence assays to measure the defects associated with F508del-CFTR allow identification of new active compounds. Br J Pharmacol 174: 525-539, 2017.

15. Bali V, Lazrak A, Guroji P, Matalon S and Bebok Z: Mechanistic approaches to improve correction of the most common diseasecausing mutation in cystic fibrosis. PLoS One 11: e0155882, 2016.

16. Basile A, Pascale M, Franceschelli S, Nieddu E, Mazzei MT, Fossa P, Turco MC and Mazzei M: Matrine modulates HSC70 levels and rescues DeltaF508-CFTR. J Cell Physiol 227: 3317-3323, 2012.

17. Verkman A, Galietta LJ V and Guy RK: Compounds having activity in increasing ion transport by mutant-cftr and uses thereof. US Patent WO2004110352. Filed July 14, 2004; issued December 23, 2004.

18. Pedemonte N,Diena T, CaciE, Nieddu E, Mazzei M, Ravazzolo R, Zegarra-Moran O and Galietta LJ: Antihypertensive 1,4-dihydropyridines as correctors of the cystic fibrosis transmembrane conductance regulator channel gating defect caused by cystic fibrosis mutations. Mol Pharmacol 68: 1736-1746, 2005.

19. Cateni F, Zacchigna M, Pedemonte N, Galietta LJ, Mazzei MT, Fossa P, Giampieri M and Mazzei M: Synthesis of 4-thiophen-2'-yl-1,4-dihydropyridines as potentiators of the CFTR chloride channel. Bioorg Med Chem 17: 7894-7903, 2009.

20. Giampieri M, Vanthuyne N, Nieddu E, Mazzei MT, Anzaldi M, Pedemonte N, Galietta LJ, Roussel C and Mazzei M: Asymmetric 4-Aryl-1,4-dihydropyridines potentiate mutant cystic fibrosis transmembraneconductance regulator (CFTR). Chem Med Chem 7: 1799-1807, 2012.

21. Galietta LJ, Haggie PM and Verkman AS: Green fluorescent protein-based halide indicators with improved chloride and iodide affinities. FEBS Lett 499: 220-224, 2001.

22. Marengo B, De Ciucis C, Ricciarelli R, Passalacqua M, Nitti M, Zingg JM, Marinari UM, Pronzato MA and Domenicotti C: PKCdelta sensitizes neuroblastoma cells to L-buthionine-sulfoximine and etoposide inducing reactive oxygen species overproduction and DNA damage. PLoS One 6: e14661, 2011

23. Colla R, Izzotti A, De Ciucis C, Fenoglio D, Ravera S, Speciale A, Ricciarelli R, Furfaro AL, Pulliero A, Passalacqua M, et al: Glutathione-mediated antioxidant response and aerobic metabolism: two crucial factors involved in determining the multi-drug resistance of high-risk neuroblastoma. Oncotarget 7: 70715-70737, 2016.

24. Galietta LV, Jayaraman S and Verkman AS: Cell-based assay for high-throughput quantitative screening of CFTR chloride transport agonists. Am J Physiol Cell Physiol 281: C1734-C1742, 2001.

25. McAuley DF and Elborn JS: Cystic fibrosis: Basic science. Paediatr Respir Rev 1: 93-100, 2000.

26. Deeks ED: Lumacaftor/Ivacaftor: A review in cystic fibrosis. Drugs 76: 1191-1201, 2016. 